\title{
Visualization of the Stress-Strain State of Shell Structures Using Virtual and Augmented Reality Technologies*
}

\author{
Alexey Semenov ${ }^{10000-0001-9490-7364]}$, \\ Iurii Zgoda 2[0000-0001-6714-500X] \\ 1 Saint Petersburg State University of Architecture and Civil Engineering, Saint-Petersburg, \\ 190005, Russia. \\ sw. semenov@gmail. com \\ 2 Saint Petersburg State University of Architecture and Civil Engineering, Saint-Petersburg, \\ 190005, Russia. \\ yurii.zgoda@mail.ru
}

\begin{abstract}
The paper describes a mathematical model of changes in the geometry of thin-shell structures for visualization of the analysis data on their stress-strain state (SSS). Based on this mathematical model, a visualization module for shell SSS visualization using VR and AR technologies was developed. The interactive visualization environment Unity 2019.3 and C\# programming language were used. The interactive visualization module makes a 3D image of a shell structure and visualizes the SSS either through heat maps over the shell or through the changes in the shell geometry on the basis of the shell type, its geometric characteristics, and SSS analysis data (transferred to the visualization module by means of a JSON file). While working on the visualization module, the authors developed a system of components that makes it possible to visualize any 3D surface with coordinate axes (including numbers with a pitch determined automatically), visualize heat maps with a graduated scale, visualize a mesh over the graph to improve the perception of the surface deformations. The middle surface can also be deformed on the basis of SSS analysis data. This solution increases the efficiency of the work of specialists in civil engineering and architecture and can be used when training specialists in courses on thin-shell structures and procedural geometry.
\end{abstract}

Keywords: Shells, Stress-Strain State, Virtual Reality, Augmented Realty, Unity.

\section{Introduction}

Thin-shell structures actively studied due to their unique properties are highly strong and stable while using the minimum amount of materials [1]. Thin-shell structures are

Copyright (C) 2020 for this paper by its authors. Use permitted under Creative Commons License Attribution 4.0 International (CC BY 4.0).

* The research was supported by RSF project № 18-19-00474. 


\section{A. Semenov, I. Zgoda}

often used in shipbuilding, aircraft and spacecraft construction, mechanical and civil engineering. Great attention is paid to studies on plates and shells since such structures are highly strong and stiff and have a variety of design shapes [2].

However, the issues of shell visualization have not been solved in full. The majority of studies on shells cover only the stress-strain state (hereinafter - the SSS) of a shell structure relative to the middle surface (Fig. 1), while deformations in the global coordinates are not considered. While such software packages as ANSYS and LIRA-SAPR enable graphic visualization of deformations, there is no standard technique or algorithm of visualization of deformed shells for variational analysis methods. In the meantime, the use of variational methods such as the Ritz method can significantly improve the accuracy of the analysis and reduce its time [3].

Informative visualization of the SSS of shells is important for their detailed study. In many cases, a researcher will examine the analysis data more efficiently if they are presented as a 3D animation rather than static contoured maps or 2D graphs.

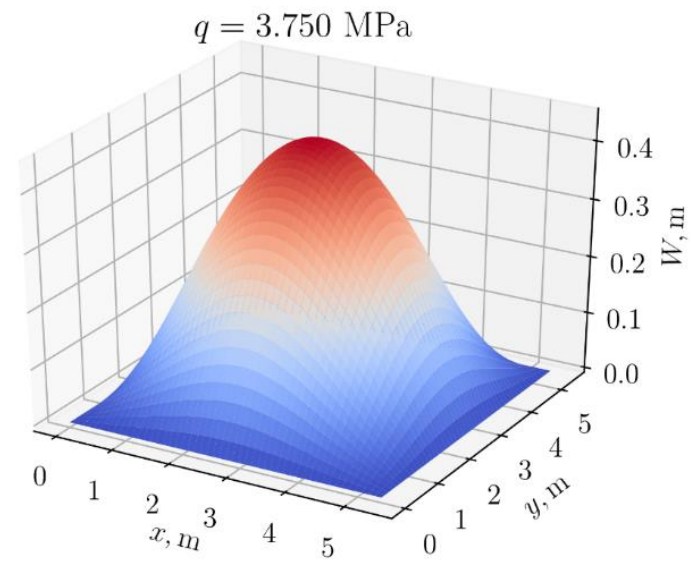

Fig. 1. Visualization of vertical displacements relative to the middle surface

VR and AR technologies are particularly important in this context [4]. Virtual reality gives a $3 \mathrm{D}$ representation of structural deformations. Augmented reality makes it possible to see the structure in the real world, which is also useful for studies on shell structures.

The purpose of this study is to develop a software package (hereinafter - the SP) for the analysis of the SSS and visualization of shell structures using VR and AR technologies

\section{SP Architecture}

The software package consists of two modules: the SSS analysis module and the visualization module. The SP architecture is shown in Fig. 2. 


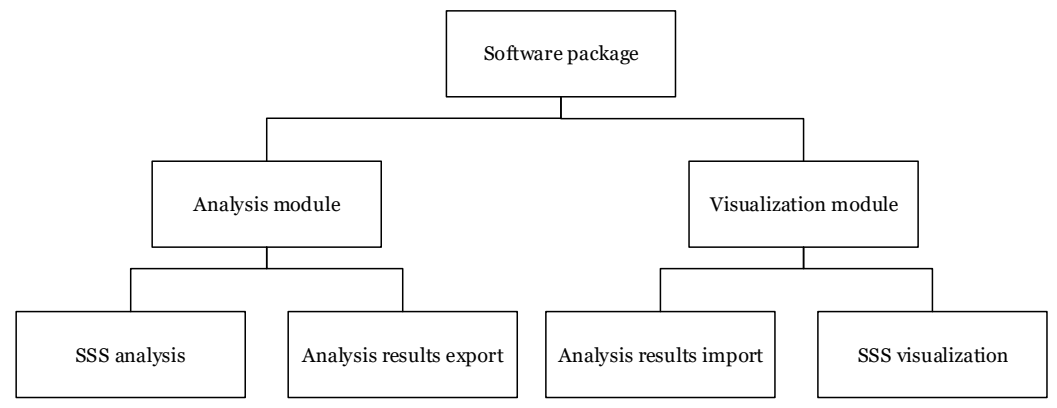

Fig. 2. Software package for shell analysis

The visualization module deserializes the file with the SSS analysis data, performs procedural generation of the geometry of the shell structure, and, then, visualizes the SSS through either heat maps presented over the shell or through changing the geometry of the shell structure.

The main feature of the described solution is the ability to render shell SSS using virtual and augmented reality technologies. At the moment, there are no solutions that allow visualizing the calculation results stored in standard formats (such as VTK) on mobile augmented or virtual reality platforms. For this reason, existing renderers (such as ParaView) couldn't be used and a custom rendering solution was developed. Visualization module imports the analysis results in custom data format used for SSS data storage in the analysis module because using other data formats doesn't provide benefits within the investigated problem.

\section{$3 \quad$ SSS of a Shell}

Analysis of the SSS of a shell structure means minimization of the functional of the total potential strain energy (which is a sum of the work of internal and external forces) on the basis of the Timoshenko (Reissner-Mindlin) model. The Ritz method is used for numerical search for the functional minimum. It reduces the variational problem to a problem of unconstrained optimization of the function of several variables. For this purpose, the required displacements functions $U(x, y), V(x, y), W(x, y)$ as well as functions of normal segment turning angles to the middle surface $\Psi_{x}(x, y), \Psi_{y}(x, y)$ are replaced with the following approximations:

$$
\begin{gathered}
U=U(x, y)=\sum_{k=1}^{\sqrt{N}} \sum_{l=1}^{\sqrt{N}} U_{k l} X_{1}^{k} Y_{1}^{l}, \quad V=V(x, y)=\sum_{k=1}^{\sqrt{N}} \sum_{l=1}^{\sqrt{N}} V_{k l} X_{2}^{k} Y_{2}^{l}, \\
W=W(x, y)=\sum_{k=1}^{\sqrt{N}} \sum_{l=1}^{\sqrt{N}} W_{k l} X_{3}^{k} Y_{3}^{l}, \\
\Psi_{x}=\Psi_{x}(x, y)=\sum_{k=1}^{\sqrt{N}} \sum_{l=1}^{\sqrt{N}} P S_{k l} X_{4}^{k} Y_{4}^{l}, \quad \Psi_{y}=\Psi_{y}(x, y)=\sum_{k=1}^{\sqrt{N}} \sum_{l=1}^{\sqrt{N}} P N_{k l} X_{5}^{k} Y_{5}^{l},
\end{gathered}
$$


where approximation functions $X_{1}^{k}-X_{5}^{k}, Y_{1}^{l}-Y_{5}^{l}$ are known and predetermined by the conditions of shell fixing, and parameters $U_{k l}-P N_{k l}$ are unknown numeric coefficients; $N_{-}$is the quantity of expansion terms.

Thus, the functional $E_{s}=E_{s}\left(U, V, W, \Psi_{x}, \Psi_{y}\right)$ is approximated by the function of several variables, and it is sufficient to use the approximation functions and the numeric coefficients' values ensuring the minimum of the functional to recover the SSS analysis data.

\section{Geometry of a Shell}

When using variational principles for making a mathematical model, the geometry of a shell structure is found through Lame parameters and principal curvatures. However, it does not seem too comfortable to make a curvilinear coordinate system to generate the geometry of a shell structure on the basis of these parameters only. Papers dealing with shell visualization [5] suggest using a parametric notation for shell structures instead.

Many shells can be described in the parametric form, which relates each of the points of the middle surface in a 2D space to a point in a 3D coordinate system. Therefore, the question of how deformations are applied to the shell middle surface should be solved. In this paper, the local basis in each point of the middle surface is used to solve this problem, which makes it possible to use displacements in the global coordinates instead of displacements $U(x, y), V(x, y), W(x, y)$ relative to the middle surface. To build the geometry of a shell with a certain thickness $h$, ratios for displacements in an arbitrary layer of the shell that follow from the Timoshenko model are used on the basis of the analysis for the middle surface.

Let us describe a parametric shell in a generalized form below. Each point of such a shell is determined through the following ratios:

$$
\left\{\begin{array}{l}
X=X(x, y), \\
Y=Y(x, y), \\
Z=Z(x, y) .
\end{array}\right.
$$

To apply deformations, i.e. displacement of the points in a horizontal, vertical and nor-

mal directions, to such a geometry, we need to find vectors

$$
N_{U}=\left(\frac{\partial X}{\partial x}, \frac{\partial Y}{\partial x}, \frac{\partial Z}{\partial x}\right),
$$
$N_{V}=\left(\frac{\partial X}{\partial y}, \frac{\partial Y}{\partial y}, \frac{\partial Z}{\partial y}\right)$

for each point of the shell, after which they should be normalized. These vectors determine the horizontal and vertical directions of displacement of the points, respectively. The vector product of these normalized vectors is the normal to the surface of the shell in the point $N_{W}(x, y)$. Analytical expressions of the basis of these vectors are derived for various types of shell structures in this paper. 
Visualization of the Stress-Strain State of Shell Structures Using Virtual and Augmented... 5

\subsection{Doubly Curved Shallow Shell}

The input parameters of a doubly curved shallow shell are linear dimensions $\mathrm{a}, \mathrm{b}$, and radii of circular arcs $R_{1}, R_{2}$. Let us introduce additional parameters $R=\max \left\{R_{1}, R_{2}\right\}-\min \left\{R_{1}, R_{2}\right\}$ and $r=\min \left\{R_{1}, R_{2}\right\}$. In this case, the parametric form for this shell will be as follows:

$$
\left\{\begin{array} { l } 
{ X = ( R + r \operatorname { c o s } x ) \operatorname { s i n } y , } \\
{ Y = ( R + r \operatorname { c o s } x ) \operatorname { c o s } y , } \\
{ Z = r \operatorname { s i n } x . }
\end{array} \quad \left\{\begin{array}{l}
x \in\left[-\frac{\min \{a, b\}}{2 r}, \frac{\min \{a, b\}}{2 r}\right], \\
y \in\left[-\frac{\max \{a, b\}}{2(R+r)}, \frac{\max \{a, b\}}{2(R+r)}\right],
\end{array}\right.\right.
$$

where $\mathrm{x}$ is the turning angle of a small radius; $\mathrm{y}$ is the turning angle of a large radius.

The expression for the basis in each point of the shell is as follows:

$$
\left\{\begin{array}{l}
N_{U}=(-\sin x \sin y,-\sin x \cos y, \cos x), \\
N_{V}=(\cos y,-\sin y, 0), \\
N_{W}=(\cos x \sin y, \cos x \cos y, \sin x) .
\end{array}\right.
$$

The middle surface of a shallow shell is given in Fig.3.

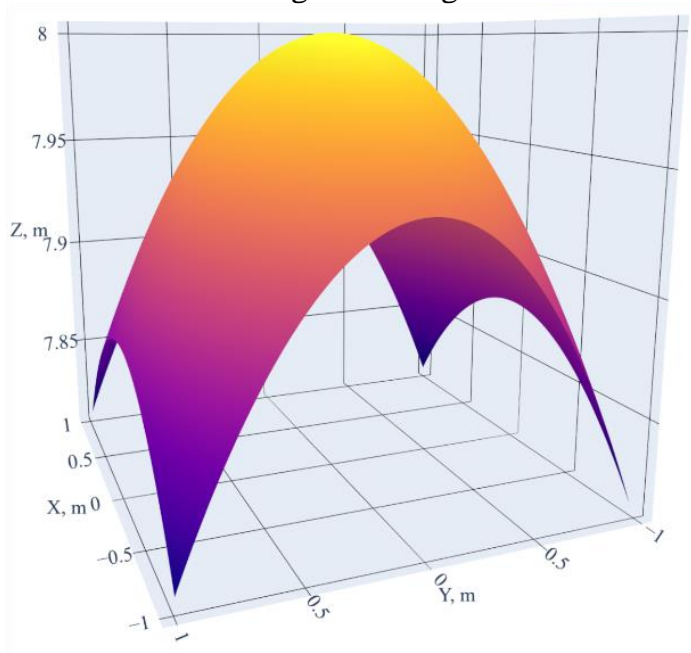

Fig. 3. Middle surface of a doubly curved shallow shell

\subsection{Spherical Shell}

The input parameters of a spherical shell are linear parameters a, a1, b and radius $\mathrm{R}$. The parametric form of a spherical shell is as follows: 


$$
\left\{\begin{array} { l } 
{ X = R \operatorname { s i n } x \operatorname { s i n } y , } \\
{ Y = R \operatorname { c o s } x , } \\
{ Z = - R \operatorname { s i n } x \operatorname { c o s } y . }
\end{array} \quad \left\{\begin{array}{l}
x \in\left[a_{1}, a\right] \\
y \in\left[-\frac{b}{2}, \frac{b}{2}\right]
\end{array}\right.\right.
$$

where $\mathrm{x}$ and $\mathrm{y}$ coincide with the latitude and longitude, respectively. The basis in the point of the middle surface is determined as follows:

$$
\left\{\begin{array}{l}
N_{U}=(\cos x \sin y,-\sin x,-\cos x \cos y), \\
N_{V}=(\cos y, 0, \sin y), \\
N_{W}=(\sin x \sin y, \cos x,-\sin x \cos y) .
\end{array}\right.
$$

The appearance of the middle surface of a spherical shell is given in Fig. 4.

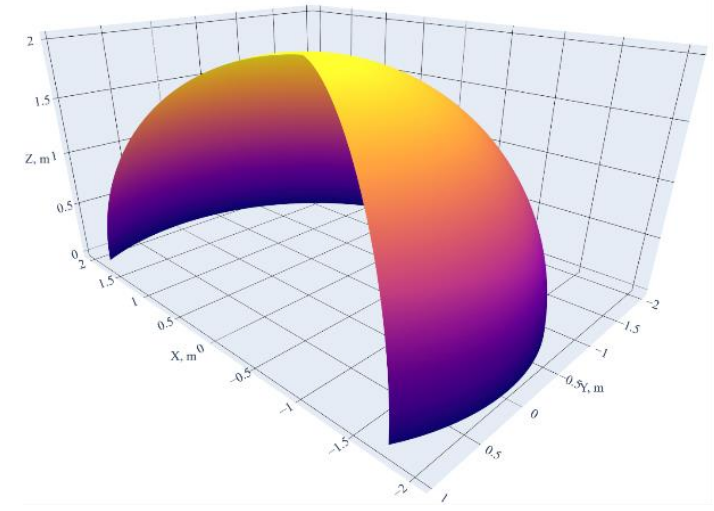

Fig. 4. Middle surface of a spherical shell

\subsection{Toroid-Shape Shell}

The model of a toroid-shape shell coincides with the model of a spherical shell but includes displacement $\mathrm{d} 1$ from the vertical axis of rotation of the sphere. The parametric form of a toroid-shape shell is as follows:

$$
\left\{\begin{array} { l } 
{ X = R \operatorname { s i n } x \operatorname { s i n } y + d _ { 1 } \operatorname { s i n } y , } \\
{ Y = R \operatorname { c o s } x , } \\
{ Z = - R \operatorname { s i n } x \operatorname { c o s } y - d _ { 1 } \operatorname { c o s } y . }
\end{array} \quad \left\{\begin{array}{l}
x \in\left[a_{1}, a\right], \\
y \in\left[-\frac{b}{2}, \frac{b}{2}\right] .
\end{array}\right.\right.
$$

The basis in the point of the middle surface is determined as follows:

$$
\left\{\begin{array}{l}
N_{U}=(\cos x \sin y,-\sin x,-\cos x \cos y), \\
N_{V}=(\cos y, 0, \sin y), \\
N_{W}=(\sin x \sin y, \cos x,-\sin x \cos y) .
\end{array}\right.
$$

The appearance of the middle surface of a toroid-shape shell is given in Fig.5. 
Visualization of the Stress-Strain State of Shell Structures Using Virtual and Augmented... 7

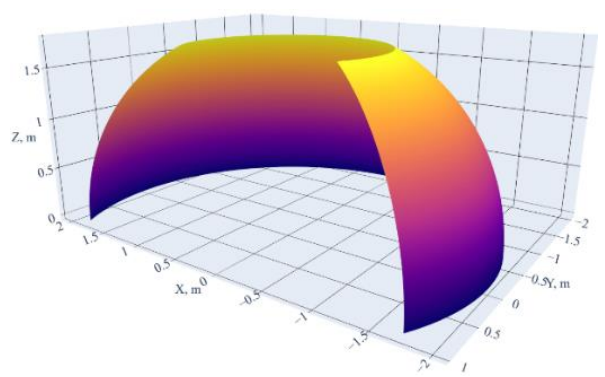

Fig. 5. Middle surface of a toroid-shape shell

\subsection{Cylindrical Shell}

The input parameters of a cylindrical shell are linear dimensions $a, a l, b$ and radius $R$. The parametric form of a cylindrical shell is as follows:

$$
\left\{\begin{array} { l } 
{ X = x - \frac { a - a _ { 1 } } { 2 } , } \\
{ Y = R \operatorname { c o s } y - R , } \\
{ Z = - R \operatorname { s i n } y . }
\end{array} \quad \left\{\begin{array}{l}
x \in\left[a_{1}, a\right], \\
y \in\left[-\frac{b}{2}, \frac{b}{2}\right]
\end{array}\right.\right.
$$

Curvilinear coordinate $x$ is along the element, while curvilinear coordinate $y$ is along the circle made by the cross-section of the cylinder with a plane parallel to its base.

The basis in an arbitrary point of the middle surface is as follows:

$$
\left\{\begin{array}{l}
N_{U}=(1,0,0), \\
N_{V}=(0,-\sin y,-\cos x), \\
N_{W}=(0, \cos y,-\sin y) .
\end{array}\right.
$$

The appearance of the middle surface of a cylindrical shell is given in Fig.6.

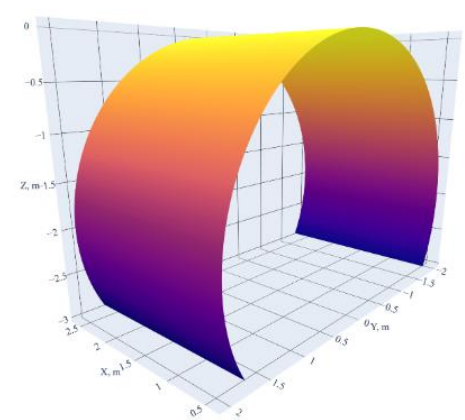

Fig. 6. Middle surface of a cylindrical shell 
The input parameters of a catenoid shell are linear dimensions $a, a l, b$ and parameter $c$. The parametric form of a catenoid shell is as follows:

$$
\left\{\begin{array} { l } 
{ X = x - \frac { a - a _ { 1 } } { 2 } , } \\
{ Y = c \cdot \operatorname { c o s h } ( x - \frac { a - a _ { 1 } } { 2 } ) \operatorname { c o s } y - c , } \\
{ Z = - c \cdot \operatorname { c o s h } ( x - \frac { a - a _ { 1 } } { 2 } ) \operatorname { s i n } y . }
\end{array} \left\{\begin{array}{l}
x \in\left[a_{1}, a\right], \\
y \in\left[-\frac{b}{2}, \frac{b}{2}\right] .
\end{array}\right.\right.
$$

The basis for such a shell takes the following form:

$$
\left\{\begin{array}{l}
N_{U}=\frac{\left(1, c \sinh \left(\frac{a-a_{1}}{2}-x\right) \cos y, c \sinh \left(\frac{a-a_{1}}{2}-x\right) \sin y\right)}{\sqrt{c^{2} \sinh ^{2}\left(x-\frac{a_{1}-a}{2}\right)+1}} \\
N_{V}=(0,-\sin y,-\cos y) \\
N_{W}=\frac{\left(c \sinh \left(\frac{a-a_{1}}{2}-x\right), \cos y,-\sin y\right)}{\sqrt{c^{2} \sinh ^{2}\left(\frac{a_{1}-a}{2}-x\right)+1}} .
\end{array}\right.
$$

The appearance of the middle surface of a catenoid shell is given in Fig.7.

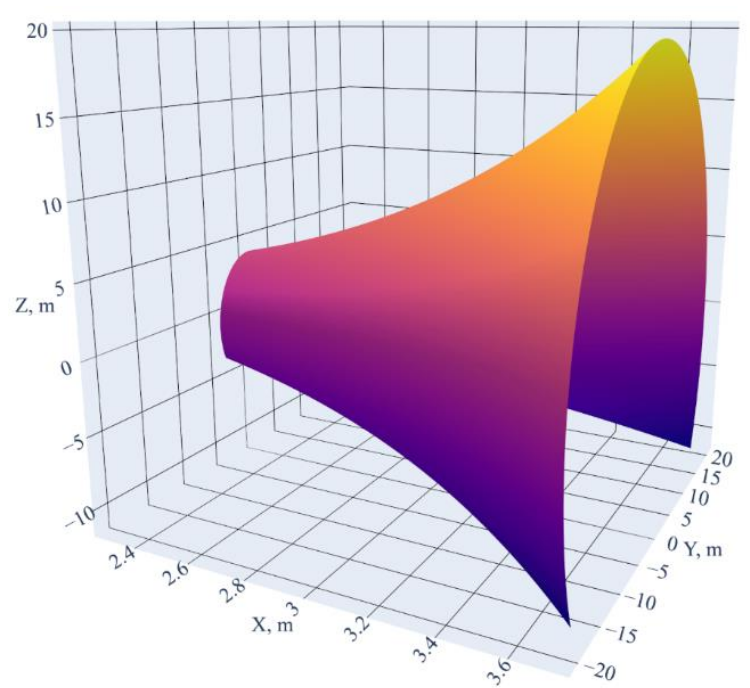

Fig. 7. Middle surface of a catenoid shell 
Visualization of the Stress-Strain State of Shell Structures Using Virtual and Augmented... 9

\subsection{Conical Shell}

The input parameters of a conical shell are linear dimensions $a, a l, b$. The parametric form of a conical shell is as follows:

$$
\left\{\begin{array} { l } 
{ X = x \operatorname { c o s } \theta - \frac { a _ { 1 } + a } { 2 } , } \\
{ Y = x \operatorname { s i n } \theta \operatorname { c o s } y , } \\
{ Z = - x \operatorname { s i n } \theta \operatorname { s i n } y . }
\end{array} \quad \left\{\begin{array}{l}
x \in\left[a_{1}, a\right], \\
y \in\left[-\frac{b}{2}, \frac{b}{2}\right] .
\end{array}\right.\right.
$$

Curvilinear coordinate $x$ is along the element, while curvilinear coordinate y is along the circle made by the cross-section of the cone with a plane parallel to its base.

The basis for a conical shell is as follows:

$$
\left\{\begin{array}{l}
N_{U}=(\cos \theta, \sin \theta \cos y,-\sin \theta \sin y), \\
N_{V}=(0,-\sin y,-\cos y), \\
N_{W}=(-\sin (\theta), \cos y \cos \theta,-\cos \theta \sin y) .
\end{array}\right.
$$

The appearance of the middle surface of a conical shell is given in Fig.8.

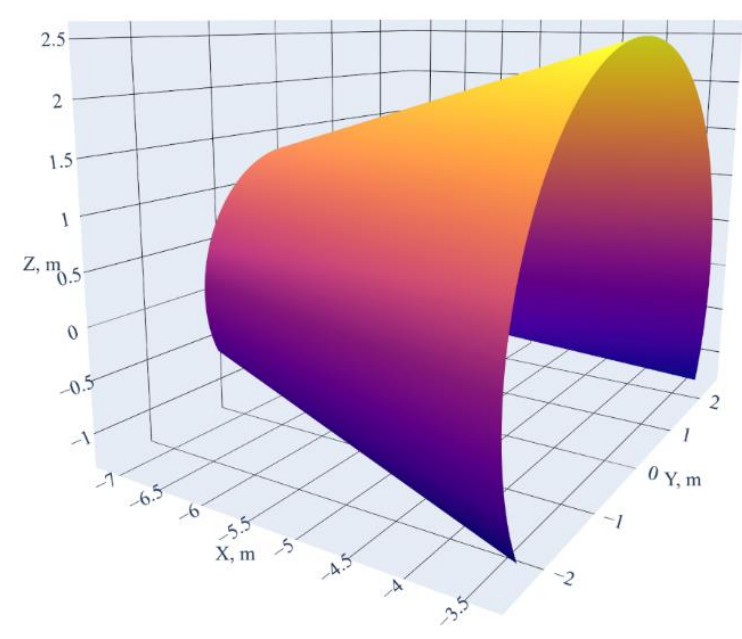

Fig. 8. Middle surface of a conical shell

\section{Shell Visualization}

To implement the shell visualization module that uses VR and AR technologies, the interactive visualization environment Unity 2019.3 and C\# programming language were used. The interactive visualization module makes a 3D image of a shell structure and visualizes the SSS either through heat maps over the shell or through the changes 
in the shell geometry on the basis of the shell type, its geometric characteristics, and SSS analysis data (transferred to the visualization module by means of a JSON file).

There is an option of using the proposed module without SSS visualization. This visualization mode can be useful to architects when they examine various forms of structures and to students studying shell structures. This allows for a better understanding of a relationship between the parameters of a shell structure and its final appearance.

While working on the visualization module, the authors developed a system of components that makes it possible to visualize any 3D surface with coordinate axes (including numbers with a pitch determined automatically), visualize heat maps with a graduated scale, visualize a mesh over the graph to improve the perception of the surface deformations. The middle surface can also be deformed on the basis of SSS analysis data.

\section{$6 \quad$ Results and Discussion}

The SP for visualization of shell structures was tested for shells of various shapes and using various visualization options. Fig.9 provides a demonstration of the SSS of a shell structure using heat maps and geometry changes.

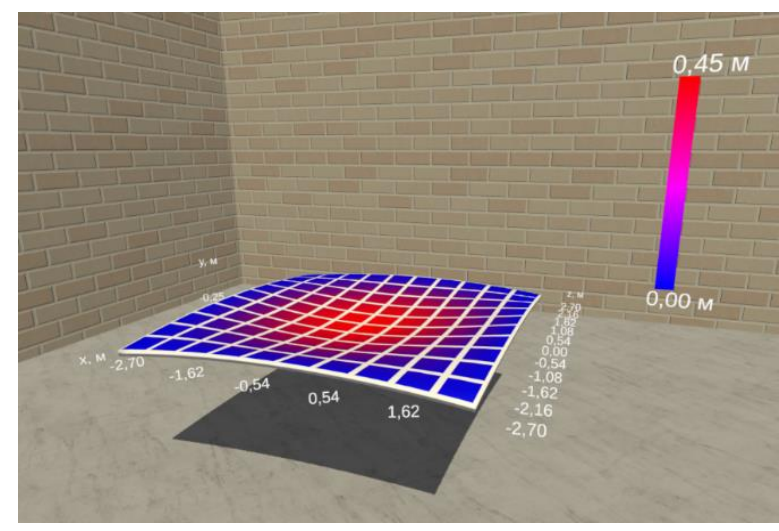

Fig. 9. Deformation of a shallow shell when exposed to a load

As follows from Fig. 9, visualization has great information capacity and describes data on the shell deformation both visually and numerically. The suggested visualization module helps to study the shell SSS in a real-world scale and with the initial proportions preserved. The clarity of such visualization in comparison to traditional visualization of the deflection relative to the middle surface (Fig. 1) is obvious.

As stated above, the suggested visualization module not only makes it possible to visualize the shell SSS but also can be used by students studying procedural modeling in architecture allowing them to clearly depict shell structures depending on their geometric parameters. Fig. 10 presents a shallow shell with lesser curvature radii and larger linear dimensions than those in Fig. 9. As you can see, a change in geometric parameters of shells of the same type can lead to significant changes in the final shape. 
Visualization of the Stress-Strain State of Shell Structures Using Virtual and Augmented... 11

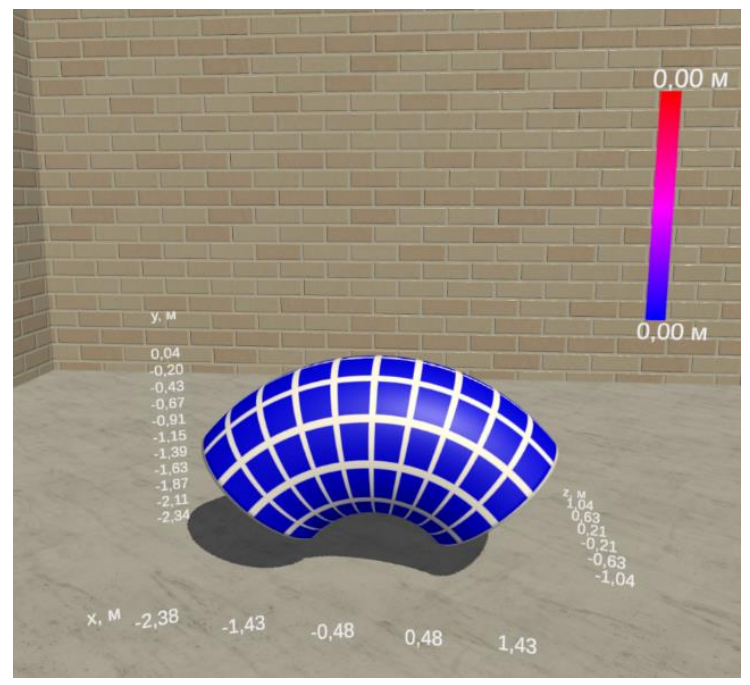

Fig. 10. Visualization of a shell with a small radius of rotation

The visualization module can be implemented using VR and AR technologies. AR visualization is demonstrated in Fig. 11.

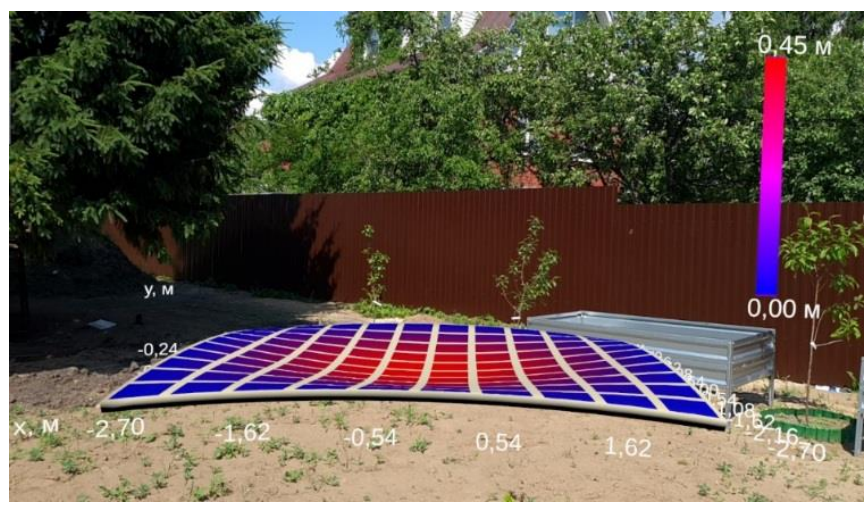

Fig. 11. AR visualization of a shell

\section{Conclusion}

Thus, the visualization module for the SSS of shell structures, enabling VR and AR visualization, was developed.

The solution can be used as a tool for informative and clear visualization of the shell SSS or when training students majoring in architecture and civil engineering in courses on thin-shell structures. 
12 A. Semenov, I. Zgoda

\section{Acknowledgments}

The research was supported by RSF (project No. 18-19-00474).

\section{References}

1. Solovei, N.A., Krivenko, O.P., Malygina, O.A.: Finite element models for the analysis of nonlinear deformation of shells stepwise-variable thickness with holes, channels and cavities. Magazine of Civil Engineering 53(1), 56-69 (2015). https://doi.org/10.5862/MCE.53.6

2. Karpov, V.V.: Strength and stability of reinforced shells of rotation. In two parts. Part 1. Models and algorithms for studying the strength and stability of reinforced shells of rotation. Fizmatlit, Moscow (2010).

3. Senjanović, Ivo, et al. Vibration analysis of rotating toroidal shell by the Rayleigh-Ritz method and Fourier series. Engineering Structures 173, 870-891 (2018).

4. Van Dam, A., Forsberg, A. S., Laidlaw, D. H., LaViola, J. J., Simpson, R. M. Immersive VR for scientific visualization: A progress report. IEEE Computer Graphics and Applications, 20(6), 26-52. (2000).

5. Aseev, A.V., Makarov, A.A., Semenov, A.A.: Visualization of the stress-strain state of thinwalled ribbed shells. Bulletin of Civil Engineers 3, 226-232 (2013). 